\title{
Correlation between L3 skeletal muscle index and prognosis of patients with stage IV gastric cancer
}

\author{
Zheng Kang ${ }^{1}$, Lingling Cheng ${ }^{2}$, Kang $\mathrm{Li}^{1}$, You Shuai ${ }^{3}$, Ke Xue ${ }^{1}$, Yuejiao Zhong ${ }^{3}$, Lingxiang Chen ${ }^{3}$ \\ ${ }^{1}$ Department of Radiology, Jiangsu Cancer Hospital and Jiangsu Institute of Cancer Research and The Affiliated Cancer Hospital of Nanjing Medical \\ University, Nanjing, China; ${ }^{2}$ Department of Medical Oncology, Yancheng Traditional Chinese Medicine Hospital Affiliated to Nanjing University \\ of Chinese Medicine, Yancheng, China; ${ }^{3}$ Department of Medical Oncology, Jiangsu Cancer Hospital and Jiangsu Institute of Cancer Prevention and \\ The Affiliated Cancer Hospital of Nanjing Medical University, Nanjing, China \\ Contributions: (I) Conception and design: Z Kang; (II) Administrative support: None; (III) Provision of study materials or patients: K Li, K Xue; (IV) \\ Collection and assembly of data: L Cheng, Y Shuai; (V) Data analysis and interpretation: Y Zhong, L Chen; (VI) Manuscript writing: All authors; (VII) \\ Final approval of manuscript: All authors. \\ Correspondence to: Yuejiao Zhong; Lingxiang Chen. Department of Medical Oncology, Jiangsu Cancer Hospital and Jiangsu Institute of Cancer \\ Prevention and The Affiliated Cancer Hospital of Nanjing Medical University, Nanjing 210009, China. Email: Zhongyuejiao1977@126.com; \\ bg4wfc@126.com.
}

Background: To explore the relationship between L3 skeletal muscle index (SMI) and the prognosis of patients with stage IV gastric cancer (GC).

Methods: A total of 27 patients with stage IV GC requiring chemotherapy admitted to our hospital from 1 April 2015 to 20 May 2019 were selected as participants. The Kaplan-Meier method was used to describe the survival time of all participants. By evaluating the L3 plane CT images, the mass index $\left(\mathrm{cm}^{2} / \mathrm{m}^{2}\right)$ of $\mathrm{L} 3$ skeletal muscle (including psoas major, erector spinae, quadratus psoas, transversus abdominis, external oblique abdominis, and internal oblique abdominis) was calculated to study the changes of L3 SMI during treatment and the correlation between L3 SMI and clinical features. The log-rank method was used to analyze the correlativity between the survival time of patients and their general data, L3 SMI, or other indicators.

Results: The survival time of 27 patients with stage IV GC was 7.4-49.9 months, with a mean survival time of 19.72 months and a median survival time of 16.17 months. The 1 -year survival rate was $77.78 \%$, and the 3-year survival rate was $7.41 \%$. During treatment, L3 SMI continued to decline in 20 of the 27 participants $(74.07 \%)$. After the first chemotherapy, 17 participants $(62.96 \%)$ met the criteria of sarcopenia syndrome, and after the fourth chemotherapy, 19 participants $(70.37 \%)$ met the criteria of sarcopenia syndrome. The L3 SMI was shown to be significantly correlated with body mass index (BMI) and Onodera's prognostic nutritional index (OPNI) (both $\mathrm{P}<0.05)$, but not with age, gender, dietary intake, and primary site (all $\mathrm{P}>0.05$ ). Log-rank test showed that there was a correlation between L3 SMI and survival time of patients $(\mathrm{P}<0.05)$. The average survival time of participants with sarcopenia syndrome (16.78 months) was significantly lower than that of those without sarcopenia syndrome $(25.58$ months $)(\mathrm{P}<0.05)$.

Conclusions: There is a significant correlation between L3 SMI and survival time, and L3 SMI can be used as a potential index to evaluate the prognosis of patients with stage IV GC.

Keywords: L3 skeletal muscle index (L3 SMI); stage IV gastric cancer (stage IV GC); prognosis

Submitted Jul 30, 2021. Accepted for publication Oct 16, 2021.

doi: 10.21037/jgo-21-556

View this article at: https://dx.doi.org/10.21037/jgo-21-556 


\section{Introduction}

Gastric cancer (GC) is one of the most common digestive tract malignant tumors in the world. There are about 1 million new GC patients worldwide every year (1). According to tumor, node, metastasis (TNM) staging, the tumor cells of stage IV GC have invaded serosa (visceral peritoneum) or adjacent structures, with high heterogeneity, and a variety of biological behaviors. Metastasis can occur in the peritoneum, liver, and lymphatic system, and the prognosis is very poor. With the development of new chemotherapeutic drugs, the median survival time of patients with stage IV GC has increased to 5-14 months. The prognosis of GC is the result of the interaction between tumor and body, and the biological behavior of tumor is the most important factor to determine the prognosis. Currently, TNM stage is the most commonly used index to evaluate the prognosis. However, it is difficult to accurately evaluate the prognosis with one or several quantitative indicators, and other potential prognostic indicators still need to be explored.

Cachexia is an internationally recognized cancer risk factor. In recent years, some researchers have proposed a new definition of cachexia, that is, malignant tumor patients with sarcopenia accompanied by weight loss of more than $2 \%$ can be classified as cancer cachexia (2). Other studies have shown that more than $50 \%$ of patients with advanced cancer suffer from varying degrees of sarcopenia (3). Although sarcopenia syndrome is not the same as cachexia, it is closely related to cancer patients, so the awareness of sarcopenia in increasing among researchers. Sarcopenia encompasses a group of complex syndromes characterized by progressive decline of whole-body skeletal muscle mass and strength, which leads to limb dysfunction, decline of quality of life,

Table 1 Baseline data of patients with stage IV GC

\begin{tabular}{lc}
\hline Item & Number of cases \\
\hline Age (years) & $60.47 \pm 10.44$ \\
Gender (male/female) & $22 / 5$ \\
Intake (moderate/severe decline) & $19 / 8$ \\
BMI (low/normal/overweight) & $5 / 15 / 7$ \\
OPNI & $41.26 \pm 6.85$ \\
Primary site & $3 / 23 / 1$ \\
(cardia/stomach/esophagogastric junction) & \\
\hline
\end{tabular}

GC, gastric cancer; BMI, body mass index; OPNI, Onodera's prognostic nutritional index. and even death. The main causes of sarcopenia are aging, chronic consumptive disease, inflammatory reaction, nutritional deficiency, malignant tumor, and so on (4). It is closely related to poor clinical outcome and short survival time. Recent studies have shown that in patients with a variety of gastrointestinal malignancies (including liver cancer, colorectal cancer, and so on), the prognosis of patients with sarcopenia is significantly worse than that of those without sarcopenia (5-7). At present, there are few studies on the relationship between sarcopenia and GC in China and internationally, and L3 skeletal muscle index (SMI) is a widely used skeletal muscle mass assessment index. Therefore, in this study, we enrolled 27 patients with stage IV GC. First, the Kaplan-Meier method was used to describe the survival time of all participants. Then, L3 SMI was calculated by evaluating the L3 plane CT images to explore the changes of L3 SMI during treatment and its correlation with clinical features. The L3 SMI levels of males and females in an epidemiological survey of gastrointestinal cancer patients conducted by Prado et al. (8) in Canada were used as the diagnostic criteria for sarcopenia syndrome. Logrank analysis was used to explore the correlation between L3 SMI or indicators and survival time, and to describe the progression-free survival (PFS). We present the following article in accordance with the REMARK reporting checklist (available at https://dx.doi.org/10.21037/jgo-21-556).

\section{Methods}

\section{General data}

Patients (27 cases) with stage IV GC who need chemotherapy admitted in our hospital from 1 April 2015 to 20 May 2019 were included as participants. Among them, there were 5 females and 22 males, aged $39-81(60.47 \pm 10.44)$ years. The remaining general data are shown in Table 1. All procedures performed in this study involving human participants were in accordance with the Declaration of Helsinki (as revised in 2013). The study was approved by Jiangsu Cancer Hospital (No. 20150218) and informed consent was taken from all the patients.

\section{Inclusion and exclusion criteria}

The inclusion criteria were as follows: patients (I) with GC, esophagogastric junction cancer, or epithelial cell origin cancer (adenocarcinoma, signet ring cell carcinoma, and so on) confirmed by cytology or histopathology; (II) who were 


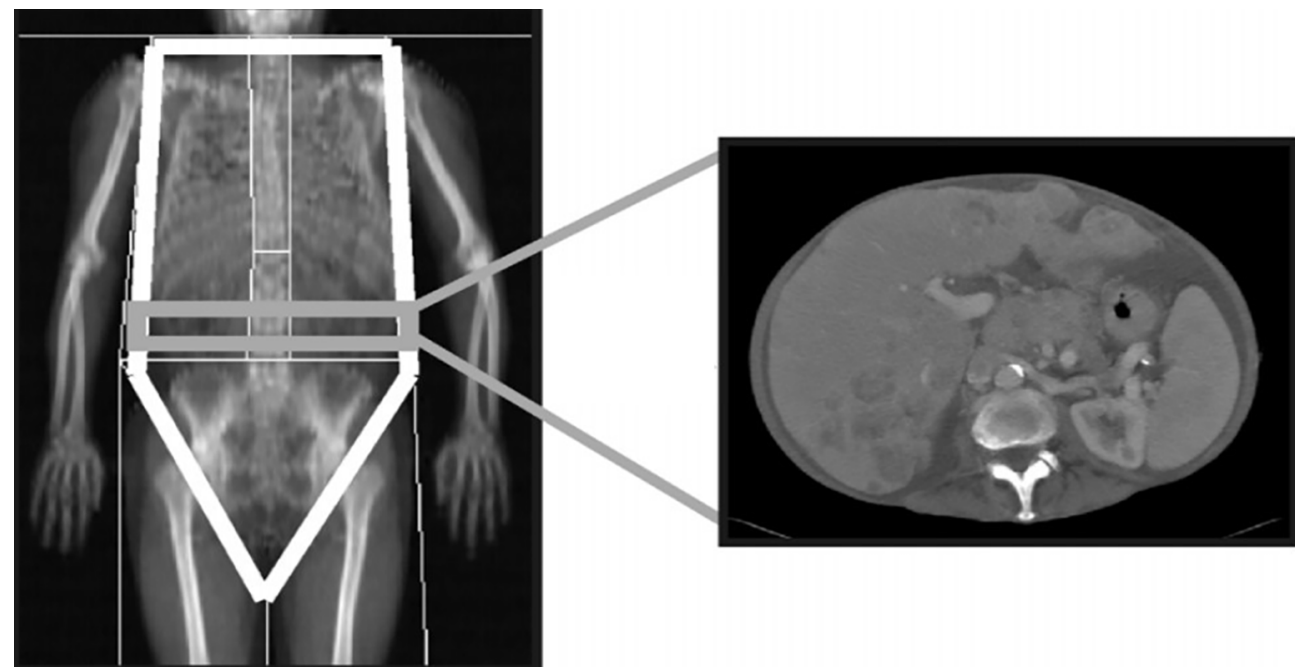

Figure 1 L3 plane capture of human body.

initially diagnosed as TNM stage IV without operation; (III) who were in good condition before treatment, with Karnofsky Performance Status (KPS) score $\geq 60$; (IV) with survival time greater than 3 months; and (V) with basically complete clinical data. The exclusion criteria were as follows: patients (I) with non-epithelial cell tumors or with other malignant tumors; (II) whose clinical stage did not conform to stage IV; (III) with severe underlying diseases of important organs; (IV) who did not agree to participate in this study.

\section{Determination of L3 SMI}

The abdominal CT images were taken with a 64-slice spiral CT machine of LightSpeed VCT series (GE Healthcare, Chicago, IL, USA). The third lumbar disc plane was selected for imaging, and two consecutive images were captured in the L3 plane. The sum of the cross-sectional areas of the skeletal muscles (including psoas major, erector spinae, quadratus psoas, transversus abdominis, external oblique abdominis, and internal oblique abdominis) was calculated. The average value of the cross-sectional area was taken, divided by the square of height to get L3 SMI $\left(\mathrm{cm}^{2} / \mathrm{m}^{2}\right)$. Male L3 SMI $\leq 52.4 \mathrm{~cm}^{2} / \mathrm{m}^{2}$ and female L3 SMI $\leq 38.5 \mathrm{~cm}^{2} / \mathrm{m}^{2}$ were used as diagnostic criteria for sarcopenia. The radiologists of our hospital worked together on a LightSpeed VCT AW4.3 workstation (Advantage Workstation, GE Healthcare) for drawing measurement, and the workstation automatically calculated the cross-sectional area of skeletal muscle (Figure 1). The L3 SMI was calculated by the following formula: L3 SMI = total skeletal muscle area of L3 cross section $/$ height ${ }^{2}\left(\mathrm{~cm}^{2} / \mathrm{m}^{2}\right)$.

\section{Other indicators}

Onodera's prognostic nutritional index (OPNI): the OPNI of all participants were calculated according to the results of blood routine and biochemical examination before treatment, and the calculation formula was OPNI = serum albumin level $(\mathrm{g} / \mathrm{L})+5 \times$ total number of lymphocytes in peripheral blood $\left(\times 10^{9} / \mathrm{L}\right)$.

The body mass index (BMI) was calculated as: weight $(\mathrm{kg}) /$ height ${ }^{2}\left(\mathrm{~cm}^{2}\right)$.

\section{Statistical analysis}

The software SPSS 17.0 (IBM Corp., Armonk, NY, USA) was used for statistical analysis. The Kaplan-Meier method was used to describe the survival time of patients with advanced GC. The log-rank method was used for univariate analysis to explore the correlativity between general data, L3 SMI, and survival time. Statistical significance was considered when $\mathrm{P}<0.05$.

\section{Results}

\section{Effect of treatment on L3 SMI}

During the treatment, 20 (74.07\%) of the 27 participants had a sustained decrease in L3 SMI. After the first chemotherapy, 

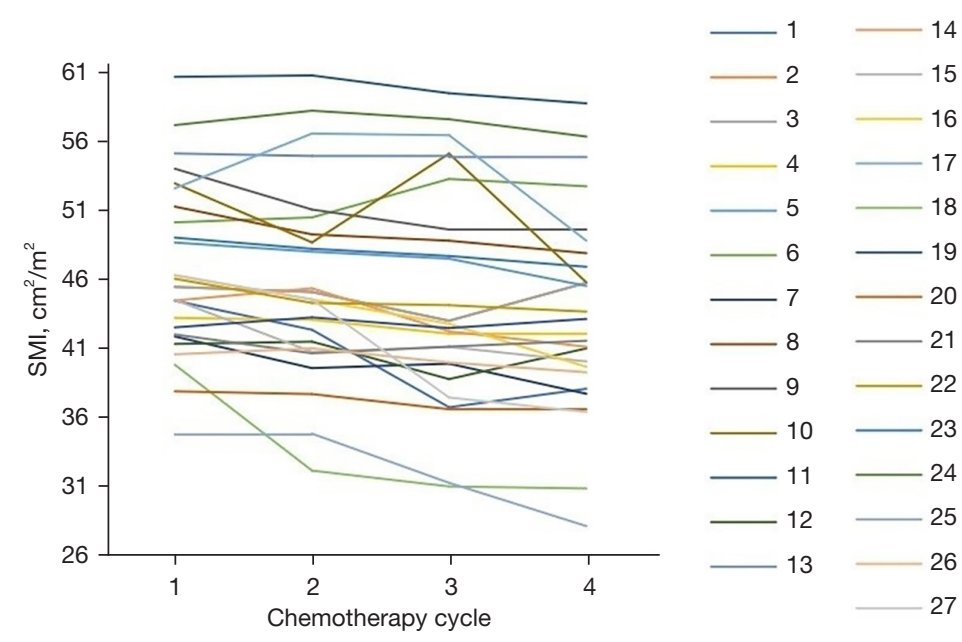

Figure 2 Changes of L3 SMI. SMI, skeletal muscle index.

17 participants $(62.96 \%)$ met the criteria of sarcopenia, and after the fourth chemotherapy, 19 participants $(70.37 \%)$ met the criteria of sarcopenia (Figure 2).

\section{Correlation between L3 SMI and clinical features}

The L3 SMI was significantly correlated with BMI and OPNI (both $\mathrm{P}<0.05$ ), but not with age, gender, dietary intake, and primary site (all $\mathrm{P}>0.05$ ) (Table 2).

\section{Overall survival (OS) of patients with stage IV GC}

In this study, the survival time of 27 participants with stage IV GC ranged from 7.4 to 49.9 months, the average survival time was 19.72 months [95\% confidence interval (CI): 15.80 to 23.64 months], and the median survival time was 16.17 months (95\% CI: 12.61 to 19.73 months). The 1 -year survival rate was $77.78 \%$, the 3 -year survival rate was $7.41 \%$, and the 5 -year survival rate was $0.00 \%$ (Figure 3).

\section{Analysis of the influencing factors of survival in stage IV GC patients}

It was revealed that $\mathrm{L} 3 \mathrm{SMI}$ was correlated with the survival time of patients $(\mathrm{P}<0.05)$, while age, gender, intake, BMI, OPNI, and primary tumor site of participants were not correlated with the survival time (all $\mathrm{P}>0.05$ ) (Table 3). After treatment, L3 SMI of participants were divided into sarcopenia group and non-sarcopenia group. The PFS of patients with sarcopenia was significantly shorter than that of patients without sarcopenia. The average survival time of participants with sarcopenia (16.78 months) was significantly lower than that of patients without sarcopenia (25.58 months) (Figure 4).

\section{Discussion}

As we all know, the OS of patients with stage IV GC is short, especially for patients with distant metastasis (such as brain metastasis, liver metastasis, lung metastasis, and so on). Due to the lack of obvious specific clinical symptoms of early GC, many have progressed to advanced stage by the time of diagnosis, and lost the opportunity of radical surgery. In recent years, comprehensive treatment for patients with stage IV GC has become a hot topic in the medical field. In this context, accurate prediction of the prognosis of such patients is very important for the selection of treatment options. At present, TNM stage, pathological type, degree of differentiation, presence of distant metastasis, metastasis site, and other prognostic factors of GC are recognized by most clinical workers, among which TMN stage plays an important role. Although these factors are very scientific and convenient prognostic indicators, the differences among individuals are huge. Even in the same stage, the clinical features, disease progression, and response to treatment are not the same. Therefore, we need to find some other reliable, economic, effective, and easily detectable prognostic indicators.

In Blauwhoff-Buskermolen's paired comparative study (9), there were significant differences between the survival curve of the high muscle loss group (9\% muscle loss), the mild muscle loss group (1.5\% to $9 \%$ muscle loss), and the 
Table 2 Correlation between SMI and clinical features

\begin{tabular}{|c|c|c|c|}
\hline Item & Number of cases & \multicolumn{2}{|c|}{$\mathrm{SMI}\left(\mathrm{cm}^{2} / \mathrm{m}^{2}\right)$} \\
\hline \multicolumn{4}{|l|}{ Age } \\
\hline$\leq 60$ years & 14 & $47.65 \pm 6.97$ & $44.88 \pm 8.29$ \\
\hline$>60$ years & 13 & $45.93 \pm 5.28$ & $42.67 \pm 6.04$ \\
\hline Gender & & 1,264 & 1,181 \\
\hline Male & 22 & $47.90 \pm 5.74$ & $44.84 \pm 6.91$ \\
\hline Female & 5 & $42.23 \pm 5.17$ & $39.29 \pm 6.34$ \\
\hline$P$ value & & 0.0731 & 0.1131 \\
\hline Severe decline & 19 & $46.59 \pm 5.73$ & $43.52 \pm 5.37$ \\
\hline$P$ value & & 0.7650 & 0.7510 \\
\hline \multicolumn{4}{|l|}{ BMI } \\
\hline Low weight & 5 & $41.83 \pm 4.11$ & $35.55 \pm 5.35$ \\
\hline Normal weight & 15 & $46.31 \pm 5.09$ & $43.37 \pm 4.80$ \\
\hline Overweight & 7 & $51.58 \pm 5.40$ & $50.66 \pm 5.26$ \\
\hline$P$ value & & $0.0095^{\star}$ & $0.0001^{*}$ \\
\hline \multicolumn{4}{|l|}{ OPNI } \\
\hline Cardia & 2 & $41.27 \pm 1.38$ & $37.06 \pm 6.19$ \\
\hline Stomach & 25 & $47.30 \pm 6.06$ & $44.35 \pm 6.94$ \\
\hline$P$ value & & 0.1867 & 0.1636 \\
\hline
\end{tabular}

*, P<0.05. SMI, skeletal muscle index; BMI, body mass index; OPNI, Onodera's prognostic nutritional index.

stable or increased muscle mass group $(<1.5 \%$ muscle loss to maximum muscle growth). These results suggest that muscle mass is correlated with the prognosis of digestive system tumors. The L3 SMI is an index used to evaluate muscle mass. It refers to the value obtained though dividing the total area of all skeletal muscles on the cross-section of L3 vertebral body obtained (by CT and MRI) by the square of height. It is highly correlated with the whole-body skeletal muscle content, and the correlation is not affected by age, gender, height, body weight, ethnicity, and so on $(10,11)$. At the same time, because it has a correlation with the prognosis of a variety of diseases, its detection is simple and easy, so it has recently become a research hotspot, especially as a prognostic factor of digestive system tumors. Low L3 SMI is an independent risk factor for poor prognosis of patients receiving chemotherapy, and has nothing to do with drug withdrawal or reduction caused by adverse drug reactions (12-14). In colorectal cancer, Miyamoto et al. (15) believe that L3 SMI is an independent risk factor for poor prognosis in patients with stage I-III colorectal cancer after radical 


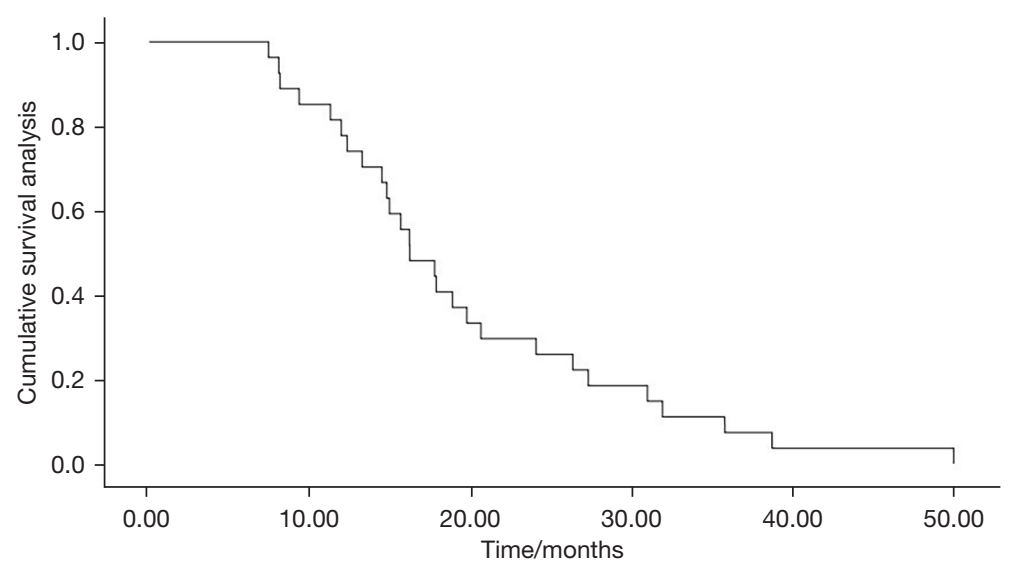

Figure 3 OS of patients with stage IV GC. OS, overall survival; GC, gastric cancer.

Table 3 Analysis of the influencing factors of survival in IV stage GC patients

\begin{tabular}{|c|c|c|c|c|}
\hline Items & $\mathrm{N}$ & Mean survival time (months) & Median survival time (months) & $P$ value \\
\hline$\leq 60$ years & 14 & $18.88(13.36-24.26)$ & $16.13(12.60-19.67)$ & \\
\hline$>60$ years & 13 & $20.77(15.02-25.18)$ & $16.17(11.13-21.20)$ & \\
\hline Gender & & & & 0.0763 \\
\hline Female & 5 & $21.94(13.17-24.39)$ & $20.90(14.57-21.28)$ & \\
\hline Intake & & & & 0.0892 \\
\hline Moderate decline & 8 & $23.69(14.92-32.48)$ & $18.82(15.57-22.01)$ & \\
\hline Severe decline & 19 & $17.23(14.01-20.46)$ & $15.61(13.04-18.16)$ & \\
\hline Normal weight & 15 & $18.03(14.38-22.62)$ & $16.13(10.08-22.18)$ & \\
\hline Overweight & 7 & $20.19(13.012-27.37)$ & $16.17(12.35-19.99)$ & \\
\hline OPNI & & & & 0.0542 \\
\hline$\leq 41.26$ & 18 & $16.73(16.66-32.76)$ & $14.90(15.59-22.01)$ & \\
\hline$>41.26$ & 9 & $24.71(13.32-20.14)$ & $18.80(12.48-17.33)$ & \\
\hline Primary site & & & & 0.9164 \\
\hline Cardia & 2 & $27.4(9.22-37.58)$ & $16.167(-)$ & \\
\hline Stomach & 25 & $19.11(15.16-22.97)$ & $16.13(12.71-19.56)$ & \\
\hline
\end{tabular}

*, P<0.05. GC, gastric cancer; BMI, body mass index; OPNI, Onodera's prognostic nutritional index; SMI, skeletal muscle index. 


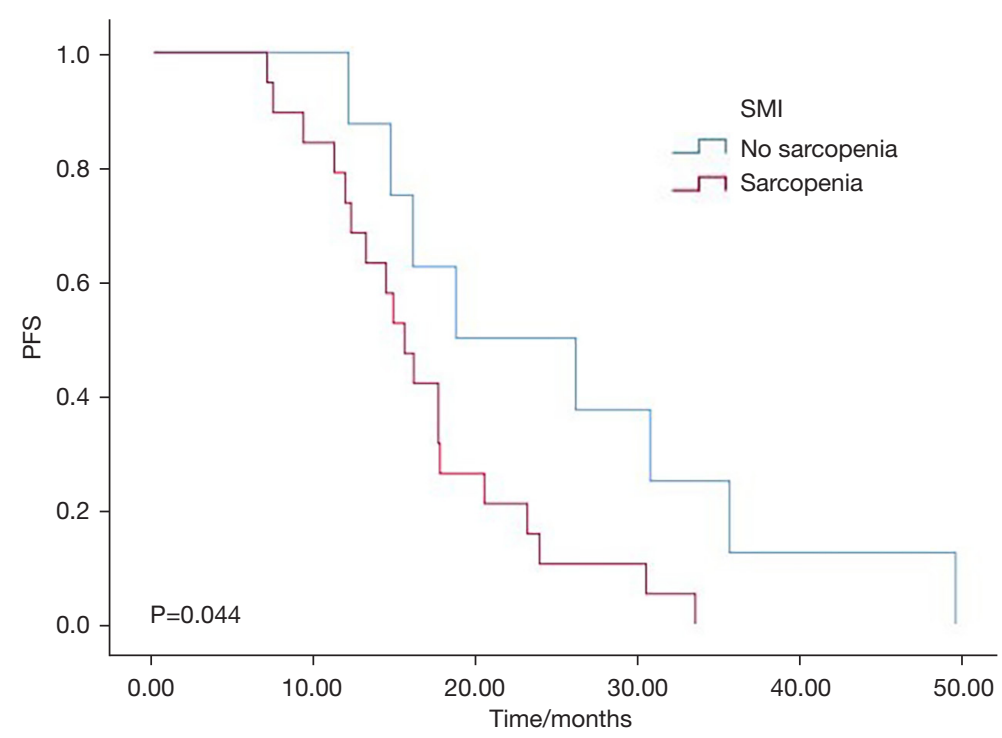

Figure 4 PFS analysis of patients with stage IV GC. PFS, progression-free survival; GC, gastric cancer; SMI, skeletal muscle index.

surgery, and its correlation with prognosis is more significant in young people. In their study, they also pointed out that the 5 -year relapse-free survival rate and OS rate of patients with low L3 SMI were $56 \%$ and $68 \%$, respectively, which were significantly lower than those with high L3 SMI. Although L3 SMI is currently frequently evaluated, in the studies by Prado et al. (8) and Go et al. (16), the proportion of patients with Eastern Cooperative Oncology Group (ECOG) score (an indicator of treatment tolerance) of 2-4 in low L3 SMI patients with sarcopenia was higher. Although Nakamura et al. (17) did not find the same statistical difference, the average value of L3 SMI of patients with an ECOG score of 2-4 was lower. The relationship between muscle mass and prognosis needs to be further verified.

In this study, most of the results were similar to the above studies. The survival curve showed that the OS time of stage IV GC patients was short. The survival time of 27 participants with stage IV GC was 7.4-49.9 months, the mean survival time was 19.72 (95\% CI: 15.80 to 23.64) months, and the median survival time was 16.17 (95\% CI: 12.61 to 19.73 ) months. The 1-year survival rate was $77.78 \%$, the 3 -year survival rate was $7.41 \%$, and the 5 -year survival rate was $0.00 \%$. By analyzing the changes of L3 SMI during the course of treatment, we found that 20 participants $(74.07 \%)$ had a continuous decrease in L3 SMI, and after the first chemotherapy, the number of participants with sarcopenia increased (from 17 to 19 cases). Further analysis of the correlation between L3 SMI and clinical features showed that L3 SMI was significantly correlated with $\mathrm{BMI}$ and OPNI (both $\mathrm{P}<0.05$ ), but not with age, gender, caloric intake, and primary site (all $\mathrm{P}>0.05)$. It is suggested that chemotherapy may reduce muscle mass, and the mass reduction is related to BMI and OPNI. Log-rank analysis showed that L3 SMI was correlated with survival time $(\mathrm{P}<0.05)$. After treatment, the L3 SMI of participants with stage IV GC were divided into a sarcopenia group and no-sarcopenia group. The PFS of participants with sarcopenia was significantly shorter than that of those without sarcopenia. The average survival time of participants with sarcopenia (16.78 months) was significantly lower than that of those without sarcopenia (25.58 months), suggesting that L3 SMI is an independent risk factor for survival.

In the clinic, due to the busy pace of daily work and the cumbersome nature of the nutrition screening procedure, most doctors will use BMI to simply assess the nutritional status of patients. However, BMI is not sufficient to evaluate the nutritional status of patients, and skeletal muscle quantification, as a new nutritional status index, can better understand the nutritional integrity of patients than the standard BMI. All patients with GC will receive abdominal CT examination, and calculating L3 SMI with CT images will not increase the economic burden of patients. Due to the different density values of each component in CT images (skeletal muscle density is usually between -29 and $+150 \mathrm{HU}$; the density values of subcutaneous tissue and visceral tissue are -190 to -30 and -150 to $-50 \mathrm{HU}$ respectively, which can perform good 
anatomical and imaging recognition of body components. Body lean body mass was assessed by calculating the skeletal muscle area in the selected layers. L3 SMI = cross-sectional area of muscle tissue at L3 level $\left(\mathrm{cm}^{2}\right) /$ height $^{2}\left(\mathrm{~m}^{2}\right)$, and L3 SMI can effectively evaluate nutritional status of patients. Another means of assessment, the ECOG score is widely used to evaluate the physical status of patients with GC. The ECOG is known to divide the physical status of patients into $0-5$ points, with a total of six levels. Its score is quantified by doctors according to patients' health function. The results are related to the doctor's experience, their understanding of patients, and other factors, which can lead to subjective results. Different from the ECOG, L3 SMI value is calculated according to the actual data of patients, and the results are not affected by subjective factors, making it more objective and powerful than the ECOG alone. We believe that the calculation of L3 SMI value after ECOG scoring in patients with GC can be icing on the cake in the evaluation of the clinical condition of patients.

In conclusion, L3 SMI can objectively evaluate the nutritional status of patients with GC, and there is a significant correlation between L3 SMI and the survival of patients. The L3 SMI is an independent risk factor affecting the survival of patients with stage IV GC, and can be used as a potential indicator for evaluating the prognosis of patients with stage IV GC.

\section{Acknowledgments}

Funding: None.

\section{Footnote}

Reporting Checklist: The authors have completed the REMARK reporting checklist. Available at https://dx.doi. org/10.21037/jgo-21-556

Data Sharing Statement: Available at https://dx.doi. org/10.21037/jgo-21-556

Conflicts of Interest: All authors have completed the ICMJE uniform disclosure form (available at https://dx.doi. org/10.21037/jgo-21-556). The authors have no conflicts of interest to declare.

Ethical Statement: The authors are accountable for all aspects of the work in ensuring that questions related to the accuracy or integrity of any part of the work are appropriately investigated and resolved. All procedures performed in this study involving human participants were in accordance with the Declaration of Helsinki (as revised in 2013). The study was approved by Jiangsu Cancer Hospital (No. 20150218) and informed consent was taken from all the patients.

Open Access Statement: This is an Open Access article distributed in accordance with the Creative Commons Attribution-NonCommercial-NoDerivs 4.0 International License (CC BY-NC-ND 4.0), which permits the noncommercial replication and distribution of the article with the strict proviso that no changes or edits are made and the original work is properly cited (including links to both the formal publication through the relevant DOI and the license). See: https://creativecommons.org/licenses/by-nc-nd/4.0/.

\section{References}

1. 1. Siegel RL, Miller KD, Jemal A. Cancer statistics, 2017. CA Cancer J Clin 2017;67:7-30.

2. Fearon K, Strasser F, Anker SD, et al. Definition and classification of cancer cachexia: an international consensus. Lancet Oncol 2011;12:489-95.

3. Fearon KC. Cancer cachexia and fat-muscle physiology. N Engl J Med 2011;365:565-7.

4. Evans WJ. Skeletal muscle loss: cachexia, sarcopenia, and inactivity. Am J Clin Nutr 2010;91:1123S-7S.

5. van Vledder MG, Levolger S, Ayez N, et al. Body composition and outcome in patients undergoing resection of colorectal liver metastases. Br J Surg 2012;99:550-7.

6. Mayanagi $S$, Tsubosa $Y$, Omae K, et al. Negative impact of skeletal muscle wasting after neoadjuvant chemotherapy followed by surgery on survival for patients with thoracic esophageal cancer. Ann Surg Oncol 2017;24:3741-7.

7. Recio-Boiles A, Galeas JN, Goldwasser B, et al. Enhancing evaluation of sarcopenia in patients with non-small cell lung cancer (NSCLC) by assessing skeletal muscle index (SMI) at the first lumbar (L1) level on routine chest computed tomography (CT). Support Care Cancer 2018;26:2353-9.

8. Prado CM, Lieffers JR, McCargar LJ, et al. Prevalence and clinical implications of sarcopenic obesity in patients with solid tumours of the respiratory and gastrointestinal tracts: a population-based study. Lancet Oncol 2008;9:629-35.

9. Blauwhoff-Buskermolen S, Versteeg KS, de van der Schueren MA, et al. Loss of muscle mass during chemotherapy is predictive for poor survival of 
patients with metastatic colorectal cancer. J Clin Oncol 2016;34:1339-44.

10. Schweitzer L, Geisler C, Pourhassan M, et al. What is the best reference site for a single MRI slice to assess wholebody skeletal muscle and adipose tissue volumes in healthy adults? Am J Clin Nutr 2015;102:58-65.

11. Taguchi S, Akamatsu N, Nakagawa T, et al. Sarcopenia evaluated using the skeletal muscle index is a significant prognostic factor for metastatic urothelial carcinoma. Clin Genitourin Cancer 2016;14:237-43.

12. Iritani S, Imai K, Takai K, et al. Skeletal muscle depletion is an independent prognostic factor for hepatocellular carcinoma. J Gastroenterol 2015;50:323-32.

13. Kamachi S, Mizuta T, Otsuka T, et al. Sarcopenia is a risk factor for the recurrence of hepatocellular carcinoma after curative treatment. Hepatol Res 2016;46:201-8.

Cite this article as: Kang Z, Cheng L, Li K, Shuai Y, Xue K, Zhong Y, Chen L. Correlation between L3 skeletal muscle index and prognosis of patients with stage IV gastric cancer. J Gastrointest Oncol 2021;12(5):2073-2081. doi: 10.21037/jgo21-556
14. Imai K, Takai K, Hanai T, et al. Skeletal muscle depletion predicts the prognosis of patients with hepatocellular carcinoma treated with sorafenib. Int J Mol Sci 2015;16:9612-24.

15. Miyamoto Y, Baba Y, Sakamoto Y, et al. Sarcopenia is a negative prognostic factor after curative resection of colorectal cancer. Ann Surg Oncol 2015;22:2663-8.

16. Go SI, Park MJ, Song HN, et al. A comparison of pectoralis versus lumbar skeletal muscle indices for defining sarcopenia in diffuse large B-cell lymphoma - two are better than one. Oncotarget 2017;8:47007-19.

17. Nakamura N, Hara T, Shibata Y, et al. Sarcopenia is an independent prognostic factor in male patients with diffuse large B-cell lymphoma. Ann Hematol 2015;94:2043-53.

(English Language Editor: J. Jones) 